\title{
THE EXISTENCE OF RATIONAL FUNCTIONS OF BEST APPROXIMATION*
}

BY

\section{J. L. WALSH}

1. Introduction. Some results have recently been proved on the approximation to arbitrary functions of a complex variable by rational functions: first, general results on the possibility of approximation with an arbitrarily small error; $\nmid$ and second, results on the degree of approximation, connecting the degree of approximation with the analytic or meromorphic character of the function approximated. $\ddagger$ In connection with both types of results, it is of interest to know that a sequence of rational functions of best approximation exists, and the present paper undertakes to prove this existence in certain cases. The term best approximation may here be interpreted for error measured in the sense of Tchebycheff, or for error measured by an integral taken in any one of several ways.

In the study of both possibility of arbitrarily close approximation and degree of approximation, much significance is attached to the position of the poles of the approximating rational functions, and consideration of such position is of central importance in the sequel.

2. Sequences of rational functions. By a rational function of $z$ of degree $n$ we mean a function which can be written in the form

$$
\frac{a_{0} z^{n}+a_{1} z^{n-1}+\cdots+a_{n}}{b_{0} z^{n}+b_{1} z^{n-1}+\cdots+b_{n}},
$$

where the denominator does not vanish identically.

THEOREM I. From any infinite family of rational functions all of the same degree $n$, there can be extracted a subsequence of functions converging continuously in the entire extended plane with the omission of at most $2 n$ points. If the limit of the sequence is not the infinite constant on the entire extended plane (except at these $2 n$ points), the limit function is a rational function of degree $n$, the convergence of the sequence is continuous in the entire extended plane with the omis-

* Presented to the Society, December 30, 1930; received by the editors January 28, 1931.

$\dagger$ W'alsh, these Transactions, vol. 31 (1929), pp. 477-502.

. Walsh, these Transactions, vol. 30 (1928), pp. 838-847.

Still further results, on interpolation and approximation by rational functions with assigned poles, are expected shortly to appear in these Transactions. 
sion of at most $n$ points, and these points are limit points of poles of the functions of the sequence.

A sequence is said to converge continuously in a region if in any closed subregion the sequence converges uniformly. In particular the limit of the sequence may be the infinite constant.

From the given family $f(z)$ (or from any infinite subsequence of it) can be extracted a new subsequence such that except in $n$ arbitrarily small circles of the plane, ${ }^{*}$ each function of the subsequence, with the possible omission of a finite number of functions, is analytic. Let a limit point of poles $\alpha_{k}^{\prime}$ of the given family be the point $\alpha^{\prime}$ :

$$
\lim _{k \rightarrow \infty} \alpha_{k}^{\prime}=\alpha^{\prime}
$$

Let functions $f_{k}^{\prime}(z)$ of the given family (or subsequence) have these respective poles $\alpha_{k}^{\prime}$. We did not require that the poles $\alpha_{k}^{\prime}$ should belong to distinct functions of the original set. This is a matter of indifference, for no function $f_{k}^{\prime}(z)$ can have more than $n$ distinct poles, and if the functions $f_{k}^{\prime}(z)$ are not all distinct functions of the original set, we can revise the sequence $f_{k}^{\prime}(z)$ if necessary and choose all the functions $f_{k}^{\prime}(z)$ distinct functions of the given set. We suppose this to be done. Let a second limit point of poles $\alpha_{i}^{\prime \prime}$ of the set $f_{k}^{\prime}(z)$ be $\alpha^{\prime \prime}$, different from $\alpha^{\prime}$ :

$$
\lim _{k \rightarrow \infty} \alpha_{k}^{\prime \prime}=\alpha^{\prime \prime}
$$

We assume, which is no restriction of generality, that the functions $f_{k}^{\prime \prime}(z)$ of the set $f_{k}^{\prime}(z)$ which have the respective poles $\alpha_{k}^{\prime \prime}$, are all distinct functions of that set. We consider further possible limit points of poles of the set $f_{k}^{\prime \prime}(z)$, and finally arrive at a subsequence $f_{k}^{(m)}(z)$ whose poles have only the limit points $\alpha^{\prime}, \alpha^{\prime \prime}, \cdots, \alpha^{(m)}$; this number $m$ is obviously not greater than $n$, for if $k$ is sufficiently large each function $f_{k}^{(m)}(z)$ has at least one pole near each point $\alpha^{(i)}$.

It is conceivable that an infinite number of functions of the given set should be constants. In this case the points $\alpha^{\prime}, \alpha^{\prime \prime}, \ldots, \alpha^{(m)}$ need not exist, but the theorem itself is obviously true. We now assume none of the functions $f_{k}^{(m)}(z)$ to be identically constant.

Let the circle $\gamma_{i}$ be a neighborhood of the point $\alpha_{i}$; we denote by $D$ the extended plane with the omission of these circles. All but a finite number of functions of the sequence $f_{k}^{(m)}(z)$ are analytic in $D$. The family $f_{k}^{(m)}(z)$ is a

* If one of the points $\alpha_{i}$ used below is the point at infinity, one of these excepted regions is a neighborhood of that point, hence the exterior of a large circle instead of the interior of a small circle. 
quasinormal family of analytic (i.e. holomorphic) functions in $D$, by virtue of the following theorem due to Montel:*

$A$ family of functions analytic in a region $D$ where they take on the value unity no more than $p$ times and the value zero no more than $q$ times is quasinormal in $D$, of order at most equal to the smaller of the integers $p$ and $q$.

The family $f_{k}^{(m)}(z)$ is quasinormal in $D$, of order not greater than $n$, no matter what may be the size of the circles $\gamma_{i}$, for each function of the family is rational of degree $n$ and can take on no value in the extended plane more than $n$ times. Hence that family is quasinormal in the entire extended plane with the exception of the points $\alpha^{\prime}, \alpha^{\prime \prime}, \ldots, \alpha^{(m)}$; this fact is readily proved by a diagonal process, with the use of variable circles $\gamma_{i}$ which approach the respective points $\alpha^{(i)}$. $\dagger$

It will be noticed that we cannot assert that we have a normal family of analytic functions in $D$. The order $n$ of the quasinormal family is actually attained, as is shown by the example

$f_{k}^{(n)}(z)=\frac{k(z+1)(z+2) \cdots(z+n)}{(z-1-1 / k)(z-2-1 / k) \cdots(z-n-1 / k)} \quad(k=1,2, \cdots)$.

Theorem I follows directly from the fact that this family $f_{k}^{(m)}(z)$ is quasinormal in the extended plane with the omission of the points $\alpha^{(i)}$. The remark that the limit function of the sequence is a rational function of degree $n$ presents no difficulty; the functions of the sequence take on no value more than $n$ times in the extended plane and therefore the limit function if not a constant can in $D$ take on no value more than $n$ times, by Hurwitz's theorem on the uniform convergence of analytic functions. The limit function is analytic at every point of the extended plane other than $\alpha^{\prime}, \alpha^{\prime \prime}, \ldots, \alpha^{(m)}$. These points are isolated singularities and none is an essential singularity, by Picard's theorem. Hence the singularities are either poles or removable singularities. This ensures the rationality of the limit function. Since no value is taken on by this function more than $n$ times, except perhaps in the points $\alpha^{\prime}, \alpha^{\prime \prime}, \cdots, \alpha^{(m)}$, the function is of degree $n$.

THEOREM II. If the moduli of an infinite family of rational functions all of degree $n$ are uniformly limited in $2 n+1$ points $\ddagger$ of the extended plane, then there

* Familles Normales, Paris, 1927, p. 67. The method of proof of Theorem I is analogous too to Montel's proof of his theorem. It is more convenient here for us to use the theory of quasinormal families of holomorphic functions than of quasinormal families of meromorphic functions.

$\dagger$ Compare Montel, loc. cit., $\S 13$.

$\ddagger$ In connection with the number $2 n+1$ which appears here as the number of points in which we suppose the given functions uniformly limited, it is of interest to mention the case $n=1$. There exists a function 
can be extracted an infinite subsequence of these functions converging continuously in the entire extended plane with the omission of $n$ points. The limit of this subsequence is a rational function of degree $n$.

We apply Theorem I directly. The limit of the subsequence cannot be the infinite constant, for if it were, the infinite constant would be the limit of the sequence at every point of the extended plane except the points $\alpha^{(i)}$ and except the possible $n$ points corresponding to the order of the sequence as a quasinormal family. The moduli of the functions of the set are uniformly limited in $2 n+1$ points, hence surely in some point of the plane different from those enumerated.*

If there is given a sequence of rational functions of degree $n$ convergent in a given region $C$, or on a point set $C$ containing at least $2 n+1$ points, the hypothesis of Theorem II is naturally satisfied. Denote by $F(z)$ the limit on $C$ of this sequence. The subsequence whose existence is ensured by Theorem II converges to some rational function $R(z)$ of degree $n$ which naturally coincides with $F(z)$ on $C$. That is to say, If a sequence of rational functions all of degree $n$ converges to some function $F(z)$ on a point set $C$ consisting of at least $2 n+1$ points, then on $C$ the function $F(z)$ is a rational function of degree $n$.

It is impossible to say here that the original sequence converges at every point of the extended plane, with possible exception of $n$ points. Indeed, let us enumerate all the points on or within the unit circle with rational coördinates: $z_{1}, z_{2}, \cdots$. The sequence

$$
\frac{1}{z-z_{1}}, \frac{\frac{1}{2}}{z-z_{1}}, \frac{\frac{1}{2}}{z-z_{2}}, \frac{\frac{1}{3}}{z-z_{1}}, \frac{\frac{1}{3}}{z-z_{2}}, \frac{\frac{1}{3}}{z-z_{3}}, \ldots
$$

converges at every point exterior to the unit circle, yet converges at none of the points $z_{1}, z_{2}, \cdots$. However, we can add the following complement to the result just proved: If the rational function $F(z)$ of degree $n$ possesses effectively $n$ poles (not neccessarily all distinct), then the given sequence converges at every point of the extended plane with the exception of these $n$ poles. The convergence is continuous in the plane so deleted.

In the general case treated above, from every subsequence of the given sequence can be extracted a new subsequence which converges on $C$ to the rational function $R(z)$ of degree $n$ and which converges continuously in the ex-

$$
w=\frac{\alpha z+\beta}{\gamma z+\delta}
$$

which takes on arbitrarily prescribed distinct values, $w_{1}, w_{2}$, $w_{3}$, in arbitrary distinct points $z_{1}, z_{2}, z_{3}$. Limitedness or convergence of a sequence of such functions in the points $z_{1}$ and $z_{2}$ implies nothing concerning limitedness or convergence in the point $z_{3}$.

* Compare Montel, loc. cit., p. 70. 
tended plane with the possible omission of $n$ points. As we change this new subsequence, the rational function $R(z)$ cannot change, for it coincides with $F(z)$ on $C$, ${ }^{*}$ but the $n$ exceptional points may vary with this subsequence; this actually occurs in the explicit example just given. However, if $R(z)$ possesses $n$ poles, each of them must be an exceptional point. If $R(z)$ possesses a multiple pole at a point $\alpha$, the poles of the functions of the subsequence which approach $\alpha$ must agree in multiplicity with $\alpha$ as a pole of $R(z)$; we discuss this fact more in detail in $\$ 4$. Hence in this case the $n$ exceptional points cannot vary with the subsequence. From every subsequence of the original sequence can be extracted a new subsequence which converges continuously to $R(z)$ in the extended plane with the omission of the poles of $R(z)$. Hence the original sequence converges in the extended plane to the function $R(z)$ except at these poles, and the convergence is continuous in this region of convergence.

The discussion we have just given of the convergence of sequences of rational functions of degree $n$ has been carried far beyond our needs for application to functions of best approximation. The results obtained can be much simplified if the poles of all the functions of the sequence lie in the same $n$ points, not necessarily all distinct (this is true, for instance, if all the functions of the sequence are polynomials, and also in the situation of $\$ 10)$. In this case convergence in $n+1$ points different from the poles implies convergence of the given sequence on the entire extended plane, except in these poles.

3. Application to Tchebycheff approximation. We are now in a position to make application of Theorem II to the problem of the existence of the rational function of best approximation.

THEOREM III. Let the function $F(z)$ be defined and continuous on a point set $C$ which is dense in itself. Suppose there exists at least one rational function $r(z)$ of degree $n$ such that $|F(z)-r(z)|$ is limited on $C$. Then there exists a rational function of degree $n$ of best approximation to $F(z)$ on $C$ in the sense of Tchebycheff.

The measure of approximation of $r(z)$ to $F(z)$ on $C$ is $\dagger$

$$
\text { bound }[|F(z)-r(z)|, z \text { on } C] \text {, }
$$

and a rational function of degree $n$ of best approximation to $F(z)$ on $C$ in the

\footnotetext{
* Two rational functions of degree $n$ which coincide in $2 n+1$ points must be identically equal, for their difference is a rational function of degree $2 n$ which vanishes in $2 n+1$ points.

- $†$ The symbol bound is intended to indicate the least upper bound of the quantity which follows it.
} 
sense of Tchebycheff is that rational function of degree $n$ or one of those rational functions for which (1) is least.

It will be noticed that the point set $C$ need not be closed. We contemplate only finite values for $F(z)$ on $C$, but in effect we allow for certain infinite values, for if on a set $C^{\prime}$ the given function $F(z)$ becomes infinite at a finite number of points, we delete those points from $C^{\prime}$ and study the approximation of the function $F(z)$ on the remaining point set $C$. The expression (1) has the same value for any rational function $r(z)$ for the two sets $C$ and $C^{\prime}$.

We proceed to the proof of 'Theorem III. By hypothesis there exists at least one rational function $r(z)$ of degree $n$ for which (1) is finite. The set of numbers, values of the expression (1) when all possible rational functions of degree $n$ are substituted for $r(z)$, has a greatest lower bound $b$. If the numbers (1) constitute merely a finite set, the theorem is obvious. In the contrary case, we choose a sequence $\left\{b_{k}\right\}$ of finite numbers (1) whose limit is $b$ :

$$
\lim _{k \rightarrow \infty} b_{k}=b .
$$

Denote by $r_{k}(z)$ a rational function corresponding to the value $b_{k}$ :

$$
\text { bound }\left[\left|F(z)-r_{k}(z)\right|, z \text { on } C\right]=b_{k} \text {. }
$$

In each point of $C$ the functions $r_{k}(z)$ are bounded, for if $b_{0}$ is the largest of the $b_{k}$ we have

$$
\begin{aligned}
\left|F(z)-r_{k}(z)\right| & \leqq b_{0}, z \text { on } C \\
\left|r_{k}(z)\right| & \leqq b_{0}+|F(z)|, z \text { on } C .
\end{aligned}
$$

It is to be remembered that we have excluded the infinite value for $F(z)$ on $C$. The set $C$, being dense in itself, contains more than $2 n$ points; the functions $r_{k}(z)$ are naturally bounded in $2 n+1$ points of $C$, so we can apply Theorem II. From the set $r_{k}(z)$ can be extracted a subsequence $r_{k}^{\prime}(z)$ converging continuously in the extended plane with the omission of $n$ points. Denote by $R(z)$ the rational function which is the limit of this sequence except possibly at these omitted points.

It is not yet possible to write

$$
\text { bound }[|F(z)-R(z)|, z \text { on } C]=b,
$$

for it is conceivable that one or more of the $n$ exceptional points considered in Theorem II should lie on $C$, and hence that the equation

$$
\lim _{k \rightarrow \infty} r_{k}^{\prime}(z)=R(z)
$$

should fail for some points $z$ on ('. Equation (3) can of course fail at no more 
than $n$ points of $C$. The left-hand member of (2) cannot be less than $b$, by the very definition of $b$.

Let us prove that the inequality

$$
\text { bound }[|F(z)-R(z)|, z \text { on } C]>b
$$

is impossible. We assume (4) to hold and reach a contradiction. Since $C$ is dense in itself, inequality (4) holding even at a single point of $C$ yields the inequality

$$
|F(z)-R(z)|>b, z \text { on } C,
$$

on an infinite subset of $C$. Hence (5) must hold at some points of $C$ where the sequence $r_{k}^{\prime}(z)$ converges to the function $R(z)$, in contradiction with the inequality $|F(z)-R(z)| \leqq b$ obtained from $\left|F(z)-r_{k}^{\prime}(z)\right| \leqq b_{k}$ by allowing $k$ to become infinite. Theorem III is now completely proved.

Theorem III can be applied to the problem (1) mentioned in $\$ 1$. Thus, ${ }^{*}$ let $M$ be a closed point set whose boundary consists of a finite number of Jordan arcs which do not separate the plane into more than a finite number of regions. If the function $f(z)$ is continuous on $M$, analytic in the interior points of $M$, there exists a sequence (not necessarily unique) of rational functions $r_{n}(z)$ of respective degrees $n$, which converges to $f(z)$ on $M$ as rapidly (approximation being measured in the sense of Tchebycheff) as does any sequence of rational functions of respective degrees $n$.

Theorem III is not true if we omit the restriction that $C$ shall be dense in itself. For let $C$ be the circle $|z|=1$ together with the origin $z=0$. Let the function $F(z)$ to be approximated be zero on the unit circle and unity in the origin. We consider approximation to $F(z)$ by rational functions of the first degree. The function $r(z)=1 /(m z+1), m>1$, approximates to $F(z)$ on $C$ (including the origin) with a maximum error $1 /(m-1)$, which approaches zero with $1 / m$. Yet there is no rational function of the first degree which approximates $F(z)$ on $C$ with a zero error. Such a function would vanish everywhere on the unit circle and hence be identically zero, but would fail to coincide with $F(z)$ in the origin.

4. Restriction of location of poles. Theorems I and II admit of interesting generalizations. In each of these theorems if the poles of the given rational functions all lie on some closed point set $E$, then the poles (if any) of the limit function also lie on $E$. This follows from the proof as already given; the points $\alpha^{(i)}$ belong to $E$. If $E$ is composed of the distinct closed point sets $E_{1}, E_{2}$, $\cdots, E$, containing respectively not more than $m_{1}, m_{2}, \cdots, m$, poles of the given rational functions, $m_{1}+m_{2}+\cdots+m_{\nu} \geqq n$, then $E_{i}$ contains not more

* Compare Walsh, loc. cit., p. 479. 
than $m_{i}$ poles of the limit function. The proof is not difficult. Let $r_{k}(z)$ be the functions of the final sequence and $r(z)$ the limit function. Only points $\alpha^{(i)}$ can be poles of $r(z)$. We prove first that the multiplicity of $\alpha^{(i)}$ as a pole of $r(z)$ is not greater than the multiplicities of the poles of the functions $r_{k}(z)$ approaching $\alpha^{(i)}$. Let $\gamma$ be a circle whose center* is $\alpha^{(i)}$ and which contains on or within it no zero of $r(z)$; if $\alpha^{(i)}$ is itself a zero of $r(z)$, nothing more needs to be proved. The circle $\gamma$ is also to be chosen so small that it contains on or within it only the one point of the set $\alpha^{\prime}, \alpha^{\prime \prime}, \cdots, \alpha^{(m)}$. We have the relation, by the uniformity of the convergence on $\gamma$,

$$
\lim _{k \rightarrow \infty}\left[\frac{-1}{2 \pi i} \int_{\gamma} \frac{r_{k}^{\prime}(z)}{r_{k}(z)} d z\right]=\frac{-1}{2 \pi i} \int_{\gamma} \frac{r^{\prime}(z)}{r(z)} d z .
$$

The right-hand member is the multiplicity of $\alpha^{(i)}$ as a pole of $r(z)$. The lefthand member is the limit of the number of poles of $r_{k}(z)$ interior to $\gamma$ diminished by the number of zeros of $r_{k}(z)$ interior to $\gamma$, which is not greater than the number of poles of $r_{k}(z)$ interior to $\gamma$. If the point $\alpha^{(i)}$ belongs to a point set $E_{j}$, the poles of $r_{k}(z)$ interior to $\gamma$ must, for $k$ sufficiently large, also belong to $E_{j}$. Hence $E_{j}$ contains not more than $m_{j}$ poles of $r(z)$. In particular if $m_{1}+m_{2}+\cdots+m_{\nu}=n$ and if the limit function has effectively $n$ poles, the function $r_{k}(z)$ must have precisely $m_{j}$ poles on $E_{j}$, for $k$ sufficiently large.

The remark we have made concerning generalizations of Theorems I and II suggests a generalization of Theorem III which is now entirely obvious. If $F(z)$ is continuous on a point set $C$ which is dense in itself and if there exists at least one admissible rational function $r(z)$ of degree $n$ such that $|F(z)-r(z)|$ is limited on $C$, then there exists an admissible rational function of degree $n$ of best approximation in the sense of Tchebycheff to $F(z)$ on $C$, that is, whose poles (if any) are restricted to lie on an arbitrary closed point set $E$.

The choice of $E$ is entirely independent of the choice of $C$. In particular if $E$ is the point at infinity, there are in Theorem I at most $n$ exceptional finite points in the plane for a sequence converging to the infinite constant and no exceptional finite points in the plane for a sequence whose limit is not the infinite constant. The limit function is naturally a polynomial of degree $n$. In Theorem II it is sufficient to require the polynomials to be bounded in but $n+1$ finite points of the plane. The case that $E$ is the point at infinity thus yields from Theorem III a proof of the well known existence of the polynomial of degree $n$ of best approximation.

More generally, we remark in connection with Theorem I that from any infinite family of rational functions all of degree $n$ and all with their poles

* The notation here assumes $\alpha^{(i)}$ to be finite. This situation can be attained by a suitable linear transformation of the complex variable if it does not present itself. 
on a closed set $E$, there can be extracted a subsequence of functions converging continuously in that part of the extended plane exterior to $E$ with the omission of at most $n$ points. If the limit of this sequence is not the infinite constant, the limit function is a rational function of degree $n$, and the convergence of the sequence is continuous on that part of the extended plane exterior to $E$. If in Theorem II we admit to consideration only rational functions with their poles on some closed point set $E$, we need only assume that the moduli of the functions of the given family are uniformly limited in $n+1$ points exterior to $E$; continuous convergence of the subsequence is assured on the extended plane exterior to $E$.

In Theorem III there need be no restriction whatever on the point set $C$ if $E$ is closed and has no point in common with $C$, provided that $C$ has at least $n+1$ points. There need likewise be no restriction on the function $F(z)$ except that it be uniformly bounded on $C$.

There is also an interesting case in which each of the point sets $E_{1}, E_{2}$, $\cdots, E_{\nu}$ considered above is a single point. We assume, as we may do with no loss of generality, that none of these points is the point at infinity. The given rational functions of Theorems I and II are of the form

$$
\begin{aligned}
r_{k}(z)=A_{0}^{(k)} & +\frac{A_{11}^{(k)}}{z-\alpha_{1}}+\frac{A_{12}^{(k)}}{\left(z-\alpha_{k}\right)^{2}}+\cdots+\frac{A_{1 m_{1}}^{(k)}}{\left(z-\alpha_{1}\right)^{m_{1}}} \\
& +\frac{A_{21}^{(k)}}{z-\alpha_{2}}+\frac{A_{22}^{(k)}}{\left(z-\alpha_{2}\right)^{2}}+\cdots+\frac{A_{2 m_{2}}^{(k)}}{\left(z-\alpha_{2}\right)^{m_{2}}} \\
& +\cdot \cdot \cdot \cdot \cdot \cdot \cdot \cdot \cdot \cdot \cdot \cdot \cdot \cdot \cdot \cdot \cdot \cdot \cdot \frac{A_{\nu m_{\nu}}^{(k)}}{\left(z-\alpha_{\nu}\right)^{m_{\nu}}},
\end{aligned}
$$

and the limit function is of the same form without the superscripts. There are in reality two different situations according as the individual numbers $m_{i}$ are prescribed, $m_{1}+m_{2}+\cdots+m_{\nu}=n$, or not. In either situation the generalization of Theorem III announces the existence of a minimizing rational function of the given form, provided that $C$ contains at least $n+1$ points and contains none of the points $\alpha_{i}$.

5. The rational function of best approximation need not be unique. It is interesting to notice that easily proved results, analogous to those mentioned in $\$ 1$, on uniform convergence of the sequence of rational functions of best approximation, on the degree of convergence of the sequence of rational functions of best approximation, and even on overconvergence, do not depend in any way on the uniqueness of the sequence of rational functions of 
best approximation, but merely on the existence of such a sequence. This remark applies not merely if best approximation is considered in the sense of Tchebycheff, but also (compare $\$ \$ 7$ and 8 ) if certain integral measures of approximation are used.

We now show by examples that the sequence of rational functions of best approximation in the sense of Tchebycheff may fail to be unique. It is not our purpose here actually to determine all Tchebycheff rational functions of best approximation for the functions to be considered. We shall content ourselves with the proof that the rational function of best approximation is not unique. Moreover, we restrict ourselves in this detailed discussion to the study of best approximation in the sense of Tchebycheff. The reader can easily study the same examples and show that the rational function of best approximation fails to be unique also if an integral measure of approximation is used.

Let us approximate to the function $F(z) \equiv z$ on the point set $C:|z|=1$. The rational functions which approximate to $F(z)$ shall be of the first degree and have their poles (if any) on the point set $E:|z|=r>2$.

If we have best approximation to $F(z)$ on $C$ by the function

$$
\frac{\alpha z+\beta}{z-r z_{0}},\left|z_{0}\right|=1,
$$

then we also have best approximation to $F(z)$ on $C$ by the function

$$
\frac{\omega \alpha z+\omega^{2} \beta}{z-\omega r z_{0}}
$$

where $\omega$ is an arbitrary quantity whose absolute value is unity. For we have

$$
\left|z-\frac{\omega \alpha z+\omega^{2} \beta}{z-\omega r z_{0}}\right|=\left|\frac{z^{2}-\omega r z_{0} z-\omega \alpha z-\omega^{2} \beta}{z-\omega r z_{0}}\right| .
$$

Substitute now $z=\omega \zeta$, so that the above expression is

$$
\left|\frac{\omega^{2} \zeta-\omega^{2} r z_{0} \zeta-\omega^{2} \alpha \zeta-\omega^{2} \beta}{\omega \zeta-\omega r z_{0}}\right|=\left|\zeta-\frac{\alpha \zeta+\beta}{\zeta-r z_{0}}\right| .
$$

The maximum of the first expression for $|z|=1$ is equal to the maximum of the last expression for $|\zeta|=1$, from which it follows that an infinity of rational functions (7) are as good an approximation to $F(z)$ on $C$ as is (6), so the latter cannot be a unique function of best approximation.

The formulas given mask one difficulty: it is conceivable that (6) should be the constant zero, in which case (7) would also be zero, and the example 
would be pointless; in every other case the two functions (6) and (7) are distinct. Rather than go through the detailed determination of a function (6) of best approximation, we merely indicate rapidly that the difficulty just raised is apparent rather than actual. The measure of approximation of the function (6) to $z$ on $C$ is the maximum of

$$
\left|z-\frac{\alpha z+\beta}{z-r z_{0}}\right|,|z|=1 .
$$

The measure of approximation of the particular function $r z /(z+r)$, whose pole lies on $E$, is the maximum of

$$
\left|z-\frac{r z}{z+r}\right|=\left|\frac{z^{2}}{z+r}\right|,|z|=1 .
$$

If $r$ is greater than 2, as we suppose, this last maximum is less than unity. Some admissible rational function which is not identically zero is therefore a better approximation to $z$ on $C$ than is the rational function zero, because the measure of approximation of the latter function to $F(z)$ on $C$ is unity. Hence the rational function whose poles lie on $E$ of best approximation to $F(z)$ on $C$ is not identically zero. The validity of the example given is now completely established.

In the example just given we might equally well have chosen for the point set $C$ the region $|z| \leqq 1$, and/or for the point set $E$ the region $|z| \leqq R>2$. There is no change to be made in the reasoning given. Or we might have chosen merely two distinct points on the circle $|z|=r>2$ as the point set $E$; again the rational function of best approximation is not unique. The example, moreover, considers explicitly approximation only by rational functions of the first degree. It can obviously be modified so as to apply to the approximation of the same function $F(z) \equiv z$ on the same point set $|z|=1$ or $|z| \leqq 1$, by rational functions of an arbitrary degree $n$ whose poles are restricted to lie on the point set $E:|z|=r>2$ or $|z| \leqq r>2$.

We give another example to show that the rational function of degree $n$ of best approximation may fail to be unique, even when the point set $E$ is the entire plane. The function $F(z) \equiv z+1 / z$ is to be approximated on the point set $C:|z|=1$ by a rational function $r(z)$ of the first degree. The rational function $r(z)$ of best approximation is not identically zero, for we have $\max [|z+1 / z|, z$ on $C]=2$, but for $r(z)=1 / z$ we have $\max [|F(z)-r(z)|$, $z$ on $C]=1$. There is no unique rational function $r(z)$ of best approximation which is identically a constant $k$, for we have by setting $w=-z$,

$$
\begin{aligned}
\max [|z+1 / z-k|, z \text { on } C] & =\max [|-z-1 / z+k|, z \text { on } C] \\
& =\max [|w+1 / w+k|, w \text { on } C],
\end{aligned}
$$


so the rational function $r(z)=-k$ is as good an approximation to $F(z)$ on $C$ as is $r(z)=+k$. There is no unique rational function of the first degree of best approximation, for we have, by setting $w=1 / z$,

$$
\max [|z+1 / z-r(z)|, z \text { on } C]=\max [|w+1 / w-r(1 / w)|, w \text { on } C],
$$

so the rational function $r(1 / z)$ is as good an approximation to $F(z)$ on $C$ as is $r(z)$. The two functions $r(1 / z)$ and $r(z)$ are distinct unless $r(z)$ is a constant; this has already been shown to be impossible.

6. A lemma on integrals. The following lemma is to be applied later, in the study of measures of approximation involving integrals. The integrals may be taken either in the sense of Riemann or Lebesgue, but in any case the functions $\phi_{k}(x)$ are naturally supposed to be integrable.

Lемма. Let $\Gamma$ be an arbitrary closed point set. If we have, for the nonnegative functions $\phi_{k}(x)$,

$$
\lim _{k \rightarrow \infty} \phi_{k}(x)=\phi(x)
$$

uniformly on every closed subset of $\Gamma$ not containing $n$ particular points of $\Gamma$, and if

$$
\lim _{k \rightarrow \infty} \int_{\Gamma} \phi_{k}(x) d x=a
$$

exists, then

$$
\int_{\Gamma} \phi(x) d x
$$

exists and is equal to or less than a.

The $n$ exceptional points of $\Gamma$ are isolated, so in order to prove the existence of (10) it is sufficient to consider a neighborhood $\gamma$ of one of those points, $P$. We assume that $\gamma$ contains no other exceptional point. Let $\gamma^{\prime}$ be a neighborhood of $P$ interior to $\gamma$. The inequality

$$
\int_{\gamma-\gamma^{\prime}} \phi_{k}(x) d x \leqq a+\epsilon,
$$

where the positive quantity $\epsilon$ is arbitrary, obviously holds for $k$ sufficiently large. Moreover, since equation (8) holds uniformly as stated, it holds uniformly in $\gamma-\gamma^{\prime}$. Hence we have

$$
\int_{\gamma-\gamma^{\prime}} \phi(x) d x=\lim _{k \rightarrow \infty} \int_{\gamma-\gamma^{\prime}} \phi_{k}(x) d x \leqq a+\epsilon,
$$

from which follows immediately the existence of 


$$
\int_{\gamma} \phi(x) d x
$$

and hence the existence of (10).

Let $\epsilon>0$ again be arbitrary. Choose the neighborhoods $\gamma^{\prime}, \gamma^{\prime \prime}, \cdots, \gamma^{(n)}$ mutually exclusive neighborhoods of the $n$ exceptional points, in such a way that

$$
\int_{\gamma^{(i)}} \phi(x) d x \leqq \frac{\epsilon}{2 n} \quad(i=1,2, \cdots, n) .
$$

There exists $K$ such that for $k>K$ we have

$$
\left|\int_{\Gamma_{-\gamma^{\prime}-\gamma^{\prime \prime}-\cdots-\gamma^{(n)}}}\left[\phi(x)-\phi_{k}(x)\right] d x\right| \leqq \frac{\epsilon}{2},
$$

for on the point set $\Gamma-\gamma^{\prime}-\gamma^{\prime \prime}-\cdots-\gamma^{(n)}$, the sequence $\left\{\phi_{k}(x)\right\}$ converges uniformly to the function $\phi(x)$. That is, we have

$$
\begin{aligned}
\int_{\Gamma} \phi(x) d x & \leqq \int_{\Gamma_{-\gamma^{\prime}-\gamma^{\prime \prime}-\cdots-\gamma^{(n)}}} \phi_{k}(x) d x+\int_{\gamma^{\prime}+\gamma^{\prime \prime}+\cdots+\gamma^{(n)}} \phi(x) d x+\frac{\epsilon}{2} \\
& \leqq \int_{\Gamma_{-\gamma^{\prime}-\gamma^{\prime \prime}-\cdots-\gamma^{(n)}}} \phi_{k}(x) d x+\epsilon .
\end{aligned}
$$

This inequality holds, once the neighborhoods $\gamma^{\prime}, \gamma^{\prime \prime}, \cdots, \gamma^{(n)}$ have been chosen, for all sufficiently large $k$. Allow $k$ to become infinite. The last integral on the right approaches a limit, by the uniform convergence of the sequence $\phi_{k}(x)$. This limit is, by (9), not greater than $a$. Hence the integral (10) is not greater than $a+\epsilon$, hence not greater than $a$.

7. Integral measures of approximation. In the integral measures of approximation now to be considered, the weight or norm function $n(z)$ [or $n(w)$ ] is to be taken as integrable, non-negative, and of course not identically zero. This last restriction avoids a trivial case; if the integrals considered are taken in the sense of Lebesgue, this restriction is naturally taken to mean that the weight function is greater than zero on some point set of measure greater than zero.

THEOREM IV. Let the function $F(z)$ be defined on the rectifiable arc, curve, or other closed point set $C$ which is linearly measurable. Suppose there exists at least one rational function $r(z)$ of degree $n$ such that

$$
\int_{C}|F(z)-r(z)|^{p} n(z)|d z|, p>0
$$


exists. Then there exists a rational function $R(z)$ of degree $n$ of best approximation, namely such that

$$
\left.\left.\int_{C} \mid F(\boldsymbol{z})-R^{\prime} \boldsymbol{z}\right)\left.\right|^{p} n^{\prime} \mathbf{z}\right)|d \boldsymbol{z}|, p>0,
$$

is not greater than the expression (11) formed for an arbitrary rational function $r(z)$ of degree $n$.

There exists, as in the proof of Theorem III, a sequence of rational functions $r_{k}(z)$ all of degree $n$ such that we have

$$
\lim _{k \rightarrow \infty} \int_{C}\left|F(\boldsymbol{z})-r_{k}(z)\right|^{p} n(z)|d z|=b,
$$

where $b$ is the greatest lower bound of all expressions of type (11). From this set $r_{k}(z)$ there can be extracted, by Theorem I, a subsequence which converges continuously in the entire plane with the omission of at most $2 n$ points. The limit of this subsequence cannot be the infinite constant, by (13); the number $b$ is of course not the infinite constant, by the hypothesis on the $r(z)$ of the theorem.

We can apply the Lemma just proved. The subsequence of the set $r_{k}(z)$ converges to a function $R(z)$, which by Theorem $\mathrm{I}$ is a rational function of degree $n$. By Theorem II and the Lemma, we now have

$$
\int_{C}|F(z)-R(z)|^{p} n(z)|d z| \leqq b .
$$

The number $b$ is the greatest lower bound of all expressions of type (11), so the inequality is excluded and the theorem is proved.

The argument just used is also valid if $C$ is an arbitrary measurable set, where the integrals are taken as surface integrals.

THEOREM V. Let the function $F(z)$ be defined on the closed measurable point set $C$ and suppose there exists at least one rational function $r(z)$ of degree $n$ such that

$$
\iint_{C}|F(z)-r(z)|^{p} n(z) d S, p>0,
$$

exists. Then there exists a rational function $R(z)$ of degree $n$ of best approximation, namely such that

$$
\iint_{C}|F(z)-R(z)|^{p} n(z) d S
$$

is not greater than the expression (14) formed for an arbitrary rational function of degree $n$. 
In Theorems IV and V we have made no mention of the location of the poles of the functions $r(z)$ of degree $n$, and these poles are naturally unrestricted as to location. Here as in Theorem III it is of course possible to admit to consideration only rational functions $r(z)$ of degree $n$ whose poles (if any) lie on an arbitrary closed point set $E$. It is still true that in each of these situations (Theorems IV and V) there exists an admissible rational function $R(z)$ of degree $n$ of best approximation; that is to say, the poles of $R(z)$, if any, also lie on $E$. Indeed, practically the whole discussion of $\$ 4$ applies here and to Theorem VI below without essential alteration.

8. An integral measure of approximation after conformal mapping. Two other cases are of interest where the measure of approximation is an integral, at least in the analogous situation of approximation by polynomials: (1) where $C$ is a simply connected region which is mapped* onto the interior of the circle $\gamma:|w|=1$ and the integral is taken over $\gamma$, and (2) where $C$ is an arbitrary closed point set whose complement $D$ is simply connected, and $D$ is mapped onto the interior or exterior of $\gamma$ and the integral is taken over $\gamma$. To be sure, the conformal map is strictly defined only in the interiors of the regions mapped, but if the correspondence is extended by continuity whenever possible to the boundaries of the regions, in the w-plane say by radial approach to $\gamma$, we still have the approximated and approximating functions defined almost everywhere on $\gamma$, so the integrals on $\gamma$ may be studied. $\dagger$ There is a possibility of complication in extending the reasoning of $\$ 7$ to both (1) and (2), for to a single point of the boundary of $C$ may correspond even an infinity of points of $\gamma$. Such a point of $C$ is a possible point of failure of the uniform convergence of the sequence or subsequence $r_{k}(z)$. The Lemma permits of failure of the uniformity of convergence in only a finite number of points of the set over which the integrals are taken. Thus the Lemma is not applicable in the most general cases (1) and (2). A detailed study of the conformal map and its implications in these general cases would lead us too far afield from the main object of the present paper, so we content ourselves with stating the following:

THEOREM VI. Let the function $F(z)$ be defined on the boundary $B$ of the simply connected region $C$ and suppose there exists at least one admissible rational function $r(z)$ of degree $n$ such that

* An arbitrary point interior to $C$ is made to correspond to the center of $\gamma$ and the rational function of best approximation depends on the particular point chosen. But approximation for an arbitrary choice of this point and an arbitrary weight function $n(w)$ is equivalent to a particular choice of this point and a suitable choice of the weight function.

A similar remark applies in case (2).

† Compare Walsh, these Transactions, vol. 32 (1930), pp. 794-816. 


$$
\int_{\gamma}|F(z)-r(z)|^{p} n(w)|d w|, p>0,
$$

exists, where the region $C$ is mapped onto the interior of the unit circle $\gamma:|w|=1$. If the region $C$ has no boundary point which corresponds to more than a finite number of points of $\gamma$, or more generally if the poles of the admissible rational functions are restricted to lie on a closed point set $E$ which contains no point of $B$ corresponding to more than a finite number of points of $\gamma$, then there exists an admissible rational function $R(z)$ of degree $n$ of best approximation, namely such that

$$
\int_{\gamma}|F(z)-R(z)|^{p} n(w)|d w|
$$

is not greater than the integral (15) formed for any other admissible rational function $r(z)$ of degree $n$.

The present application of the Lemma is not essentially different from the previous ones, and will not be given in detail. One minor difference may be noted, namely that the Lemma requires uniform convergence on certain point sets, and in the present case we can assert merely uniform convergence with the possible exception of sets of measure zero. This does not of course essentially alter the validity of the application.

Theorem VI requires only that $F(z)$ should be defined on the boundary of $C$, and approximation is in reality measured only on this boundary. It ordinarily occurs, however, that we are interested in studying the convergence of the approximating sequence not merely on the boundary but also in a region or on a more general point set. Two distinct interpretations are possible here, as mentioned above, according to whether we consider further convergence (1) on $C$ or (2) on the complement of $C$. That is to say, in effect we consider (15) as a measure of approximation (1) on $C$ or (2) on the complement of $C$. These two cases are quite distinct when one studies approximation by polynomials or approximation by rational functions whose poles are restricted to lie on closed point sets $E$ which lie (1) on the complement of $C$, or (2) in $C$, but the two cases merge into a single case when one considers approximation by rational functions with no restrictions on their poles.

So far as the present writer is aware, there has never been studied the problem of overconvergence of sequences of rational functions of best approximation, where the poles are restricted to lie on a closed point set $E$ and where the measure of approximation is taken as in (11), (14), or (15). Overconvergence can be studied in all of these cases, however, by the methods already used elsewhere for polynomials and for more general rational functions.*

\footnotetext{
* See the preceding reference and also the references given in $\$ 1$.
} 
Our methods of proof of the existence of rational functions of best approximation apply, it may be added, to many measures of approximation other than those we have considered.

9. Approximation with auxiliary conditions. A variation of the problems of approximation already considered is to require that the rational function of best approximation (considered in any one of the several ways) shall take on prescribed values in certain points $\alpha_{i}$. This requirement may be interpreted as restricting certain derivatives of the approximating functions in the points $\alpha_{i}$ if the latter are not taken all distinct:

$$
R\left(\alpha_{i}\right)=m_{i}, R^{\prime}\left(\alpha_{i}\right)=m_{i}^{\prime}, \cdots, R^{\left(k_{i}\right)}\left(\alpha_{i}\right)=m_{i}{ }^{\left(k_{i}\right)} \quad(i=1,2, \cdots, k) .
$$

The points $\alpha_{i}$ may or may not belong to $C$. The discussion we have given for the various measures of approximation is not valid in the present case, for one of the exceptional points of convergence in Theorem II may be a point $\alpha_{i}$. The equation $r_{k}\left(\alpha_{i}\right)=m_{i}$ does not in this case imply $\lim _{k \rightarrow \infty} r_{k}\left(\alpha_{i}\right)=m_{i}$. It is true, however, that all the exceptional points in Theorem II are limit points of poles of rational functions of approximation, and if we restrict those poles so that they lie on a closed point set $E$ which contains none of the points $\alpha_{i}$, the auxiliary conditions satisfied by the functions $r_{k}(z)$ are also satisfied by the limits of those functions. This follows from the uniformity of the convergence of the sequence or subsequence $\left\{r_{k}(z)\right\}$ in the neighborhood of each point $\alpha_{i}$.

Let us state the theorem resulting from this discussion if the Tchebycheff measure of approximation is used. The other theorems are so similar that they are readily supplied by the reader.

If the function $F(z)$ is defined and continuous on a point set $C$ which is dense in itself, we consider approximation to $F(z)$ on $C$ by rational functions $r(z)$ all of degree $n$ which satisfy the prescribed auxiliary conditions

$$
\begin{aligned}
& r\left(\alpha_{i}\right)=m_{i}, r^{\prime}\left(\alpha_{i}\right)=n_{i}^{\prime}, \cdots, \\
& r^{\left(k_{i}\right)}\left(\alpha_{i}\right)=m_{i}{ }^{\left(k_{i}\right)}
\end{aligned} \quad(i=1,2, \cdots, k),
$$

and whose poles lie on some closed point set $E$ which contains none of the points $\alpha_{i}$. If there exists at least one admissible function* $r(z)$ such that $|F(z)-r(z)|$ is limited on $C$, then there exists an admissible function $R(z)$ of best approximation, namely such that

$$
\overline{\text { bound }}|F(z)-R(z)|, z \text { on } C \text {, }
$$

exceeds no expression of the same form where $R(z)$ is replaced by any other admissible function.

* The existence of rational functions satisfying auxiliary conditions is considered in some detail in $\$ 10$. 
If we are willing to require that $E$ shall have no point in common with $C$, we do not need to assume here that $C$ is dense in itself, but merely, as in the comment on Theorem III, that $C$ contains more than $n$ points. Even this number may be lessened by the number of points $\alpha_{i}$ not belonging to $C$. The function $F(z)$ need merely be uniformly limited on $C$.

The theorem itself is not true if we omit the requirement that $E$ shall contain none of the points $\alpha_{i}$. We illustrate this by a simple example similar to the example already used in $\S 3$. Let us approximate to the function $F(z) \equiv 0$ on the point set $C:|z|=1$ by rational functions $r(z)$ of the first degree which satisfy the single auxiliary condition $r(0)=1$. The measure of approximation is

$$
\overline{\text { bound }}|r(z)|, z \text { on } C \text {. }
$$

The lower limit of this measure of approximation, considered for all admissible functions $r(z)$, is zero; indeed, the function $r(z)=1 /(m z+1), m>1$, is admissible, yet the upper bound of its modulus on $C$ is $1 /(m-1)$, which approaches zero with $1 / m$. There naturally exists no rational function $r(z)$ of the first degree which satisfies the auxiliary conditions whose least upper bound on $C$ is zero. Such a function, being zero on $C$, would vanish identically and could not satisfy the auxiliary condition.

10. Uniqueness of rational functions with preassigned poles. There is one case, interesting for the applications, where the rational function of best approximation not only exists but is unique.

If the rational function of degree $n$ is required to be of the form*

$$
\begin{aligned}
r_{n}(z)=A_{00} & +\frac{A_{11}}{z-\alpha_{1}}+\frac{A_{12}}{\left(z-\alpha_{1}\right)^{2}}+\cdots+\frac{A_{1 m_{1}}}{\left(z-\alpha_{1}\right)^{m_{1}}} \\
& +\frac{A_{21}}{z-\alpha_{2}}+\frac{A_{22}}{\left(z-\alpha_{2}\right)^{2}}+\cdots+\frac{A_{2 m_{2}}}{\left(z-\alpha_{2}\right)^{m_{2}}} \\
& +\cdot \cdot \cdot \cdot \cdot \cdot \cdot \cdot \cdot \cdot \cdot \cdot \cdot \cdot \cdot \cdot \\
& +\frac{A_{\nu 1}}{z-\alpha_{\nu}}+\frac{A_{\nu 2}}{\left(z-\alpha_{\nu}\right)^{2}}+\cdots+\frac{A_{\nu m_{\nu}}}{\left(z-\alpha_{\nu}\right)^{m_{\nu}}}, \\
& n=m_{1}+m_{2}+\cdots+m_{\nu},
\end{aligned}
$$

* There are two distinct points of view regarding rational functions of form (16), namely (i) to require that $r_{n}(z)$ should be of the form (16) where $n$ is prescribed and the individual numbers $m_{i}$ are not prescribed, although the numbers $m_{i}$ may be subjected to restrictions $m_{i} \leqq m_{i}{ }^{\prime}, i=1,2, \cdots, \nu$, where the $m_{i}{ }^{\prime}$ are prescribed, $n<m_{1}{ }^{\prime}+m_{2}{ }^{\prime}+\cdots+m_{\nu}{ }^{\prime}$; (ii) to require that $r_{n}(z)$ should be of form (16), where $n$ is prescribed together with each individual number $m_{i}, n=m_{1}+m_{2}+\cdots+m_{\nu}$. In $\S 4$ it is shown that in both of these cases (i) and (ii) there exists an admissible rational function $r_{n}(z)$ of best approximation. Only in case (ii) can it be proved that there exists a unique rational function of best approximation; compare the examples given in $\$ 5$. 
where the numbers $\alpha_{1}, \alpha_{2}, \cdots, \alpha_{\nu}, m_{1}, m_{2}, \cdots, m_{\nu}$ are prescribed but the numbers $A_{i j}$ are not prescribed, and if the continuous function $F(z)$ is approximated on a closed point set $C$ containing none of the points $\alpha_{i}$ but containing more than $n$ points, then the rational function of best approximation is unique.

We shall proceed to prove the truth of this theorem, whether best approximation is considered in the sense of Tchebycheff, or in the sense of least weighted $p$ th powers $(p>1)$ measured by a line or surface integral, with or without conformal mapping. The theorem is also true in each of these cases, as we shall prove, for sufficiently large $n$ even if we have prescribed auxiliary conditions relating to points distinct from the $\alpha_{i}$, provided that if approximation is measured in the sense of Tchebycheff the auxiliary conditions are of the form $r_{n}\left(\beta_{i}\right)=\left(F \beta_{i}\right)$ for all points $\beta_{i}$ belonging to $C$. This equation need not be taken to imply $r_{n}^{\prime}\left(\beta_{i}\right)=F^{\prime}\left(\beta_{i}\right)$ in case two or more of the $\beta_{\mu}$ fall together in $\beta_{i}$ on $C$.

A case which is clearly admissible, though not covered by a literal reading of (16), is that of prescribed poles at infinity; it is no loss of generality in the proof to assume all of the points $\alpha_{i}$ finite, for that situation can be obtained by a suitable linear transformation of the complex variable.

In connection with the results to be proved, it is necessary to know that under certain conditions there exists at least one function of the form (16) satisfying given auxiliary conditions. Let us show that there exists a unique function of type (16) which takes on prescribed values $B_{\mu}$ in $\rho=n+1$ prescribed points $\beta_{\mu}$ distinct from the points $\alpha_{i}$. We give the proof under the hypothesis that the points $\beta_{\mu}$ are all distinct, but the result holds in the more general case that two or more of them coincide. The condition is then naturally interpreted to mean the prescription of $r_{n}(z), r_{n}^{\prime}(z), \cdots, r_{n}^{(k)}(z)$ in the points $\beta_{\mu}$ concerned, the number $k$ depending on the number of points $\beta_{\mu}$ which are considered to coincide.

The existence and uniqueness of the function $r_{n}(z)$ depends on the solution of $\rho$ obvious linear equations for the numbers $A_{i j}$, and this solution depends on the non-vanishing.of the determinant

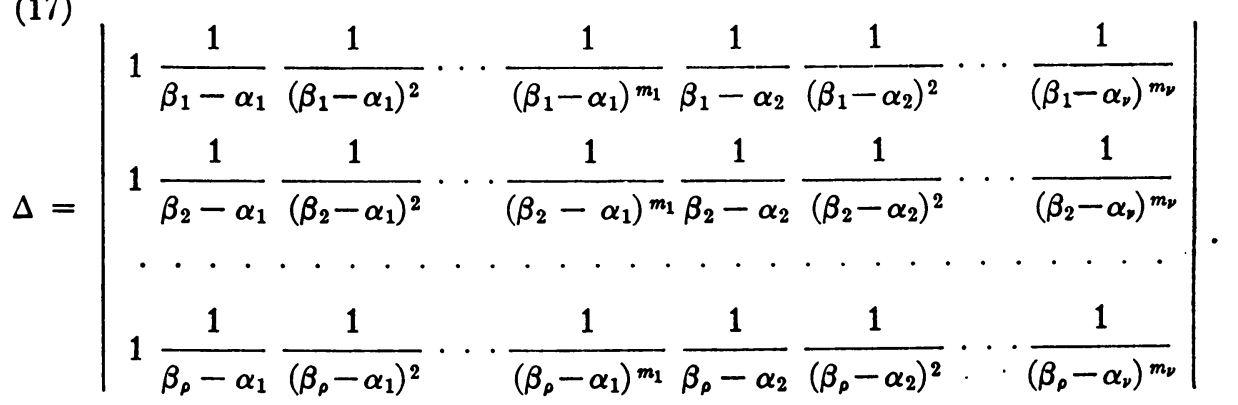


The vanishing of $\Delta$ is a necessary and sufficient condition that the homogeneous system of equations (corresponding to $B_{\mu}=0$ ) for the determination of the numbers $A_{i j}$ should have a non-trivial solution. The vanishing of $\Delta \mathrm{im}$ plies then the existence of a rational function of degree $n$ which is not identically zero and which has at least $n+1$ zeros, in the points $\beta_{\mu}$; this is impossible. Thus we have $\Delta \neq 0$, and the proof is complete.

The result just proved is of interest in the present connection as showing the existence of at least one admissible function $r_{n}(z)$, satisfying the given auxiliary conditions in $\rho$ points $\beta_{\mu}$ different from the $\alpha_{i}, \rho \leqq n+1$. Under these conditions, it follows from $\S \S 3,4,7,8,9$ that there exists at least one admissible function $r_{n}(z)$ of best approximation. We now prove further: The uniqueness of the function $r_{n}(z)$ of best approximation, with or without auxiliary conditions, follows directly from the inequality

$$
\left[\frac{|\alpha|+|\beta|}{2}\right]^{p}<\frac{1}{2}\left[|\alpha|^{p}+|\beta|^{p}\right], p>1, \alpha \cdot \beta \neq 0,
$$

if best approximation is measured by an integral as previously considered. For if there exist two distinct admissible rational functions $r_{n}(z)$ and $r_{n}^{\prime}(z)$ of best approximation to the given function $F(z)$, then $\left(r_{n}(z)+r_{n}^{\prime}(z)\right) / 2$ is actually an admissible function of better approximation:

$$
\begin{aligned}
\int n(z)\left|F(z)-\frac{r_{n}(z)+r_{n}^{\prime}(z)}{2}\right|^{p} d s< & \frac{1}{2} \int n(z)\left|F(z)-r_{n}(z)\right|^{p} d s \\
& +\frac{1}{2} \int n(z)\left|F(z)-r_{n}^{\prime}(z)\right|^{p} d s \\
= & \int n(z)\left|F(z)-r_{n}(z)\right|^{p} d s,
\end{aligned}
$$

which is of course impossible. The proof holds whether the integral be a line integral or surface integral; the numbers $\alpha$ and $\beta$ to which the inequality is applied must be different from zero on a point set of measure greater than zero. If $r_{n}(z)$ and $r_{n}^{\prime}(z)$ both satisfy given auxiliary conditions, so also does $\left(r_{n}(z)+r_{n}^{\prime}(z)\right) / 2$, and the proof is valid even if auxiliary conditions are prescribed.

Our proof of the uniqueness of the rational function of best approximation with or without auxiliary conditions is now complete in the case that approximation is measured by an integral. In order to complete the proof of the theorem already stated it remains for us to study further the case that approximation is measured in the sense of Tchebycheff: 
Let $F(z)$ be an arbitrary function continuous on a closed point set $C$, which is distinct from the points $\alpha_{1}, \alpha_{2}, \cdots, \alpha_{\mu}$ and contains at least $n+1$ points. ${ }^{*}$ Then there exists a unique rational function $r_{n}(z)$ of form (16) of best approximation to $F(z)$ on $C$ in the sense of $T$ chebycheff with preassigned auxiliary conditions

$$
r_{n}\left(\beta_{i}\right)=B_{i} \quad(i=1,2, \cdots, k),
$$

where the points $\beta_{i}$ are distinct from the $\alpha_{j}$, provided $n \geqq k-1$ and provided that $B_{i}=F\left(\beta_{i}\right)$ if $\beta_{i}$ is a point of $C$.

The case $k=0$ is not excluded here and simply means the omission of auxiliary conditions. In case the points $\beta_{i}$ are not all distinct, equation (18) is interpreted to define not merely $r_{n}\left(\beta_{i}\right)$ but also one or more derivatives of $r_{n}(z)$ at the multiple points $\beta_{i}$, depending on the number of points $\beta_{i}$ which coincide; the number $k$ is intended to denote the number of equations (18), not the number of distinct points $\beta_{i}$. The equation $B_{i}=F\left(\beta_{i}\right)$, required to hold if $\beta_{i}$ is a point of $C$, is not intended necessarily to restrict the values of any derivatives of $r_{n}(z)$, even if $\beta_{i}$ is a point of $C$. $\dagger$

We have already proved the existence of at least one admissible function of best approximation. The proof of the uniqueness may be carried out by the original method due to Tonelli and used by him in the case that $r_{n}(z)$ is a polynomial and $k=0$, although the details for the present case follow closely a modification of Tonelli's proof recently given by the present writer. $\ddagger$ The complete proof can easily be constructed by the reader. Our preliminary result on the existence and uniqueness of functions of type (16) which take on prescribed values in prescribed points is needed. Moreover, the theorem (with or without auxiliary conditions) can be extended to include even Tchebycheff approximation with a norm function, that is, the determination of a rational function $r_{n}(z)$ of form (16) such that the maximum value of

$$
n(z)\left|F(z)-r_{n}(z)\right|, z \text { on } C,
$$

* It is sufficient here to require that the set of points $C$ plus the $\beta_{i}$ contains at least $n+1$ points. Moreover, if $n$ is sufficiently large it is allowable that some of the points $\alpha_{i}$ should coincide with points $\beta_{j}$ and even that some points $\alpha_{i}$ should lie on $C$ if a sufficiently large number of those points do not lie on $C$.

$\dagger$ Indeed, if we denote by $\epsilon_{n}$ the maximum error $\left|F(z)-r_{n}(z)\right|, z$ on $C$, for the best approximation $r_{n}(z)$ (with the given auxiliary conditions) to $F(z)$ on $C$ in the sense of Tchebycheff, it is sufficient to require $\left|B_{i}-F\left(\beta_{i}\right)\right|<\epsilon_{n}$ instead of $B_{i}=F\left(\beta_{i}\right)$.

The equation $B_{i}=F\left(\beta_{i}\right)$ or some other restriction is actually necessary for the uniqueness of the function of best approximation, as is shown by the case $n=1, \alpha_{1}=\infty, C: 0 \leqq z \leqq 1, F(z) \equiv 1$, with the auxiliary condition $r_{1}(0)=0$.

$\ddagger$ These Transactions, vol. 32 (1930), pp. 335-390, \$10. 
is least; here $n(z)$ is supposed positive and continuous on $C$. This proof too follows closely the original proof due to Tonelli.* In our previous work in the present paper we did not trouble to consider Tchebycheff approximation with a norm function. The introduction of such a function throughout the paper offers not the slightest difficulty.

* Compare Walsh, these Transactions, vol. 32 (1930), pp. 794-816; p. 797, foot note.

HARVARD UNIVERSITY,

Cambridge, Mass. 